\title{
INCIDÊNCIA E CARACTERÍSTICAS ENDOSCÓPICAS DE LESÕES DAS VIAS AÉREAS ASSOCIADAS À INTUBAÇÃO TRAQUEAL EM CRIANÇAS
}

Andréa Maria Gomes Cordeiro*, Shieh Huei Shin, Iracema de Cássia Oliveira ferreira Fernandes, Albert Bousso, Eduardo Juan Troster

Trabalho realizado na Unidade de Terapia Intensiva Pediátrica do Hospital Universitário da USP, São Paulo, SP.

RESUMO - OBjetivo. Descrever a incidência e características endoscópicas de lesões das vias aéreas em crianças submetidas à intubação.

Métodos. Durante o período de dois anos (outubro/99 a outubro/0I) foi conduzido estudo prospectivo no qual todo paciente intubado, excetuando-se aqueles que evoluíram para óbito e recém-nascidos (RN) com peso inferior a $1.250 \mathrm{~g}$, foi submetido à endoscopia respiratória na extubação. Achados endoscópicos foram classificados em leves, moderados ou graves. Descriçōes foram realizadas por meio de proporções e medianas, comparações feitas por teste qui-quadrado para proporções.

Resultados. Foram estudados 6I RN e 154 crianças. Em $89,8 \%$ dos pacientes, sendo $55 \mathrm{RN}$ e 138 crianças $(p=0,89)$, foi detectada pelo menos uma lesão somando 507. Pacientes com lesões leves corresponderam a 54,8\% (IC95\%: 48,I-6I,5), aqueles com lesões moderadas foram 24,2\% (IC95\%: 18,5$30,0)$ enquanto as graves ocorreram em $10,7 \%$ dos pacientes
(IC95\%: 6,6-14,8). Locais principalmente acometidos foram glote ( $48,1 \%$ das lesões) e subglote ( $34,9 \%$ das lesões). Erosões foram as mais incidentes em ambos os grupos etários $(p=0,88)$. Edema de prega vocal foi a principal lesão moderada em ambos os grupos $(p=0,96)$, seguida por ulcerações $(p=0,92)$. Nódulos fibrosos em pregas vocais e sinéqüias foram as principais lesões graves em ambos os grupos etários $(p=0,12)$. Estenose subglótica foi detectada em $2,8 \%$ da população sem diferença entre as faixas etárias $(p=0,35)$.

ConclusöEs. Verificou-se elevada incidência de lesões em vias aéreas, sem diferença significante entre os grupos etários com relação à incidência e características das lesões. Houve predomínio de lesões leves, lesões na glote e caracterizadas por erosões, edema e ulcerações.

Unitermos: Intubação intratraqueal. Criança. Recém-nascido. Lesão das vias aéreas. Estenose subglótica

\section{INTRODUÇÃO}

Lesões de via aérea secundárias a trauma decorrente da intubação começaram a ser descritas à medida que o procedimento passou a ser realizado com maior freqüência, particularmente com a popularização desse método como alternativa ou predecessor da traqueotomia, e conseqüentemente para suporte prolongado da via aérea, no início dos anos $50^{\prime}$. Os relatos iniciais se limitavam a estudos de autópsia que descreveram diferentes tipos de lesões como ulceração e necrose de cartilagem da traquéia, edema na laringe e traquéia em adultos ${ }^{2}$. Perda completa ou focal do epitélio foi detectada na mucosa que recobre os processos vocais e região posterior da cricóide após intubação por apenas I a 3 horas; ulceração em processos vocais e subglote após 12 a 48 horas de intubação e úlceras profundas e acometimento de cartilagem (pericondrite, necrose e

\footnotetext{
* Correspondência:

Rua Jacques Felix, 96 - apt. 94

Cep: 04509-000 - São Paulo - SP
}

exposição) após 48 horas de permanência do tubo na via aérea em adultos ${ }^{3}$. Estudos em crianças mostraram resultados similares. Striker et al. reportaram os achados de autópsia em 32 crianças submetidas à intubação traqueal e descreveram edema em laringe $(50,0 \%$ das autópsias) e traquéia (53,1\%), ulceração na laringe $(28,1 \%)$ e traquéia $(34,4 \%)$ e erosão em laringe $(12,5 \%)$ e traquéia $(25 \%)^{4}$.

O mecanismo fisiopatológico primário da lesão decorrente da permanência do tubo na via aérea envolve a pressão exercida na mucosa pelo tubo ${ }^{5,6}$. O mesmo se apóia e exerce uma pressão na porção posterior da laringe afetando três sítios principais: a superfície medial das cartilagens aritenóides, aspectos mediais das junções cricoaritenóides e processo vocal; a comissura posterior na região interaritenóide e a subglote envolvendo a superfície interna da cartilagem cricóide geralmente a lâmina posterior. Devido à natureza tangencial do contato entre o tubo e a laringe a área de contato é pequena. Uma vez que quanto menor a área sobre a qual uma força é aplicada maior será a pressão resultante (pressão = força/área), altas pressões podem resultar de forças relativamente pequenas ${ }^{5}$. A pressão exercida pelas paredes firmes do tubo superou largamente a pressão de perfusão da mucosa, estimada em $30 \mathrm{mmHg}$ e alcançou $400 \mathrm{mmHg}$ em modelo animal ${ }^{7}$, dando início a um processo inflamatório local ${ }^{6}$. A resolução desse processo pode acarretar graves seqüelas na via aérea tais como estenose, sinéquias, formação de granulomas, entre outras.

Estudos baseados em avaliação endoscópica das lesões de via aérea em crianças submetidas à intubação endotraqueal têm enfocado a incidência de estenose subglótica provavelmente pela elevada morbidade associada (necessidade de traqueotomia, dilatação endoscópica ou cirurgia de reconstrução da laringe) $)^{8-13}$. No entanto, lesões graves como a estenose subglótica se desenvolvem a partir de lesões fundamentais como ulcerações e exposição de cartilagem. Existe uma seqüência de eventos que culminaria com a seqüela em via aérea ${ }^{5,6}$. Portanto, a prevenção de lesões graves requer o conhecimento da incidência de outras formas de lesão que têm o potencial de 
evolução para formas graves. Estudos de incidência das diferentes formas de lesão da via aérea associadas à intubação traqueal se limitaram à faixa etária neonatal ${ }^{14,15}$ ou envolveram pequeno número de pacientes pediátricos ${ }^{16-20}$. O presente estudo teve como objetivo descrever numa coorte de crianças submetidas à intubação traqueal a incidência e características endoscópicas de lesões de via aérea.

\section{Métodos}

Durante o período de outubro de 1999 a outubro de 200 I todo paciente admitido na Unidade de Terapia Intensiva Pediátrica e Neonatal (UTIP/UTIN) do Hospital Universitário da Universidade de São Paulo (HUUSP) que tenha necessitado de intubação traqueal foi submetido à endoscopia respiratória no momento da extubação. Foram excluídos os recém-nascidos de peso inferior a $1.250 \mathrm{~g}$ e aqueles que evoluíram ao óbito previamente à extubação.

$\mathrm{Na}$ população de estudo foram descritas e analisadas as seguintes características: idade (até 28 dias de vida ou idade superior a 28 dias), sexo, peso, escore de risco de óbito (PRISM- Pediatric Risk of Mortality para aque-


intubação, principais diagnósticos de admissão e indicações de intubação de acordo com a falência ou insuficiência orgânica principal.

O exame endoscópico foi realizado por um dos pesquisadores (AMGC). Uma amostra não-aleatória de 50 exames foi registrada em vídeo e os resultados confrontados com outro observador que desconhecia os diagnósticos estabelecidos pelo primeiro, visando aferir a concordância entre eles. Para realização do exame endoscópico foi adotado um protocolo de anestesia e todos os pacientes tiveram a saturação de oxigênio, freqüência cardíaca e pressão arterial monitorizados durante todo 0 procedimento.

Os achados endoscópicos foram classificados de acordo com proposta de Benjamin (1993) que definiu o aspecto endoscópico, a topografia e possibilidade evolutiva das lesões supraglóticas, glóticas e subglóticas (Quadro I).

A partir dessa classificação adotamos a seguinte terminologia:

- Lesões com potencial de evolução para resolução foram denominadas leves e incluíram edema (exceto os citados abaixo), hiperemia e erosão.

\begin{tabular}{|c|c|c|}
\hline \multicolumn{3}{|c|}{ Quadro I-Alterações endoscópicas pós-extubação e prognóstico (Adaptado de Benjamin ${ }^{6}$ ) } \\
\hline Tipo de alteração & Aspecto endoscópico & Possível evolução \\
\hline $\begin{array}{l}\text { Precocee } \\
\text { inespecífica }\end{array}$ & $\begin{array}{l}\text { Hiperemia } \\
\text { Edema } \\
\text { Erosão }\end{array}$ & Resolução \\
\hline Edema & $\begin{array}{l}\text { Protusão da mucosa ventricular } \\
\text { Edema prega vocal } \\
\text { Edema subglótico }\end{array}$ & $\begin{array}{l}\text { Resolução } \\
\text { Edema crônico de Reinke } \\
\text { Obstrução subglótica }\end{array}$ \\
\hline Tecido de granulação & $\begin{array}{l}\text { "Línguas" a partir do processo vocal } \\
\text { das aritenóides }\end{array}$ & $\begin{array}{l}\text { Resolução } \\
\text { Granuloma } \\
\text { Nódulo fibroso } \\
\text { Sinéquia interaritenóide }\end{array}$ \\
\hline Ulceração & $\begin{array}{l}\text { Depressões ulceradas } \\
\text { Ulceração anular em glote posterior } \\
\text { Ulceração subglótica com acometimento } \\
\text { cricóide } \\
\text { Laceração }\end{array}$ & $\begin{array}{l}\text { Sulcos cicatriciais } \\
\text { Sinéquila da glote posterior } \\
\text { Estenose subglótica } \\
\text { Cicatriz }\end{array}$ \\
\hline Miscelânea & $\begin{array}{l}\text { Sangramento } \\
\text { Deslocamento de aritenóide } \\
\text { Perfurações } \\
\text { Ulceração da cricóide }\end{array}$ & $\begin{array}{l}\text { Hematoma } \\
\text { Fixação da junção } \\
\text { cricoaritenóide } \\
\text { Infecção ou formação } \\
\text { de abscesso } \\
\text { Fístula }\end{array}$ \\
\hline
\end{tabular}

- Lesões com probabilidade de evolução para seqüelas foram denominadas lesões moderadas e incluíram edema subglótico, edema de pregas vocais, ulceração, línguas de tecido de granulação, laceração, hematoma, deslocamento de aritenóide, exposiçãa de cartilagem e depressões ulceradas.

- Seqüelas de intubação foram denominadas lesões graves e incluíram estenoses, sinéquias, paresia ou paralisia de prega vocal, granuloma, nódulos fibrosos, sulcos cicatriciais e fixação da junção cricoaritenóide. Lesões situadas abaixo da subglote não foram classificadas quanto à gravidade uma vez que não estavam inclusas na classificação proposta por Benjamin ${ }^{6}$ e adotada no estudo.

Reavaliação endoscópica foi realizada apenas para os pacientes que necessitaram de reintubação. Para esses foram registrados apenas os novos achados endoscópicos.

Consentimento para participação na pesquisa foi obtido para todos os pacientes juntamente aos pais ou representantes legais.

A descrição das características populacionais e das lesões foi realizada por meio de proporções e medianas. Comparações, quando pertinentes, foram realizadas por meio de teste qui-quadrado para proporções. Foram consideradas diferenças significantes quando o valor de p foi inferior a 0,05.

\section{Resultados}

Durante o período de estudo foram admitidos 1.014 pacientes na UTIP e 42 I na UTIN. Um total de 1.435 pacientes compôs a populaçãa da unidade nesse período. Desses, 313 pacientes $(21,8 \%)$ foram submetidos à intubação traqueal. Foram excluídos $58 \mathrm{pa}$ cientes ( 35 que evoluíram a óbito, 12 por não consentimento e II que apresentavam peso inferior a $1.250 \mathrm{~g}$ ). Em 40 pacientes não foi possível a realização do exame (oito por impossibilidade de obtenção do consentimento, 14 por dificuldades operacionais, 10 por extubação acidental e oito pacientes que foram transferidos ainda intubados).

$\mathrm{Na}$ Tabela I são descritas as principais características dos 215 pacientes estudados. A maior parte foi representada por pacientes fora do período neonatal $(71,6 \%)$. Nesse grupo houve predomínio de lactentes $(60,5 \% \mathrm{da}$ população). Pacientes do sexo masculino predominaram em ambos os grupos, 57,4\% para os abaixo de 28 dias e $55,2 \%$ para os maiores de 28 dias. Os valores do escore PRISM ${ }^{21}$ mostraram que a população acima de 28 dias, à qual se aplica, apresentava baixo risco de morte à admissão. Não houve diferença entre as faixas etárias com relação à duração da intubação $(p=0,19)$. 


\begin{tabular}{|c|c|c|}
\hline \multicolumn{3}{|c|}{ Tabela I-Características da população de estudo ( 215 pacientes) } \\
\hline \multirow[t]{2}{*}{ Variável } & \multicolumn{2}{|c|}{ Mediana(Variação) } \\
\hline & $\leq 28 \mathrm{~d}(6 \mathrm{I})$ & $>28 d(154)$ \\
\hline Idade & $5,4 d(\mid-26)$ & 4 meses (I mês - I4 anos) \\
\hline Peso* & $2.700(1.250-4.600)$ & $6,2(2,1-54,0)$ \\
\hline PRISM• & NA & $12(3-29)$ \\
\hline Risco de morte (\%) • & NA & $9,0(2,2-64,5)$ \\
\hline Duração da intubação em dias & $4,4(\mid-63 d)$ & $5,0(10 h-53 d)$ \\
\hline
\end{tabular}

*Peso em gramas para $\leq 28$ dias, peso em Kg para > 28 dias; Avaliados em 102 pacientes (excluídos 61 recém-nascidos, 10 pacientes c/ shunt intracardíaco, 15 c/ doença pulmonar crônica e 27 pacientes com dados incompletos); h- horas, d- dias; NA- não se aplica

\section{Gráfico I- Distribuição percentual das 507 lesões por faixa etária e topografia SUPGL - supraglote, GL - glote, SUBGL - subglote, VAI - via aérea inferior}

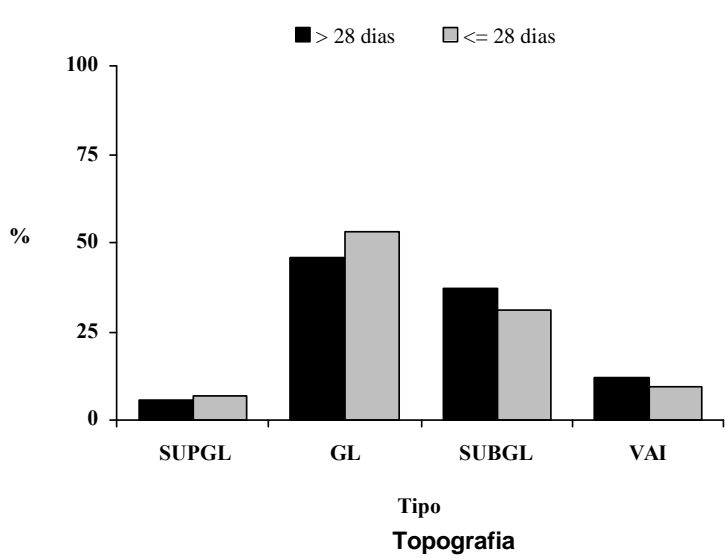

SUPGL-supraglote, GL-glote, SUBGL-suglote, VAl-via aérea inferior

Os diagnósticos principais à admissão foram classificados, de acordo com o sistema acometido, em problemas respiratórios, cardiovasculares, neurológicos e outros. Foram detectados 504 diagnósticos principais, sendo 377 na população com idade acima de 28 dias e 127 nos recém-nascidos. Doenças de origem respiratória prevaleceram em crianças maiores (56,5\% dos diagnósticos) e também entre recém-nascidos (54,3\% dos diagnósticos) $(p=0,67)$. Ao contrário, a doença neurológica foi a condição menos comum em ambos os grupos: $6,6 \%$ em crianças maiores e 7,9\% em recém-nascidos $(p=0,63)$. Broncopneumonia/pneumonia foram os principais diagnósticos de origem respiratória nos 154 pacientes maiores $(53,9 \%)$. Por outro lado, entre os recém-nascidos, responderam por apenas 22,9\% dos diagnósticos de admissão $(p<0,00 I)$. Nesses a doença de membrana hialina foi a doença respiratória mais prevalente tendo ocorrido em 37,7\% dos recém-nascidos.
A intubação foi indicada por insuficiência respiratória na maior parte dos pacientes (57,8\% dos pacientes acima de 28 dias de vida e $73,8 \%$ dos recém-nascidos). Instabilidade neurológica foi raramente indicação de intubação nessa população, tendo ocorrido em cinco pacientes maiores que 28 dias (3,3\%) e dois recém-nascidos (3,3\%). Comparando os dois grupos etários com relação às indicações de intubação, notamos diferenças estatisticamente significantes apenas para a insuficiência respiratória que prevaleceu nos recém-nascidos $(p=0,04)$.

\section{Estudo endoscópico}

Exame endoscópico normal foi observado em apenas 10,2\% (22/215) dos pacientes dentre os quais seis eram recém-nascidos e 16 eram crianças maiores que 28 dias $(p=0,89)$. Nos demais $89,8 \%$ da população estudada (IC 95\%: 85,8 - 93,8) sendo 55 recém-nascidos e 138 crianças maiores que 28 dias de vida, foi detectada pelo menos uma lesão na via aérea. Um grupo de 55 pacientes necessitou de reintubação sendo 39 dentre as 154 crianças maiores que 28 dias de vida (25,3\%), e 16 dentre 6 I recém-nascidos $(26,2 \%)(P=0,97)$. Em alguns foram necessárias até três reintubações e 83 procedimentos de reintubação foram conduzidos.

Foram detectadas 507 lesões, sendo 358 em 138 crianças maiores que 28 dias de vida e 149 lesões em 55 recém-nascidos. Portanto foram identificadas em média 2,6 e 2,7 lesões por criança maior que 28 dias de vida e recémnascido, respectivamente. A distribuição topográica e por faixa etária das lesões mostrou que a glote (48, I\% das lesões; IC 95\%: 43,9 - 52,3) e subglote (34,9\% das lesões; IC 95\%: 30,8 - 39,0) foram os locais principalmente acometidos em ambos os grupos. Lesões em via aérea inferior foram incomuns (I I,0\% das lesões; IC 95\%: 8,3 - 13,7) assim como em supraglote (5,9\% das lesões; IC 95\%: 3,8-8,0). Não houve diferença estatisticamente significante entre os grupos etários com relação às lesões em glote $(p=0, \mid$ I) e subglote $(p=0,2 I$ ) (Gráfico I).

Como houve com freqüência a associação de lesões em um mesmo paciente apresentaremos o percentual de pacientes com lesões de gravidades distintas. Para tal levamos em consideração a presença de pelo menos uma lesão grave, pelo menos uma lesão moderada ou apenas lesões leves. Observamos que 54,8\% dos pacientes (IC 95\%: 48,I - 6I,5) apresentaram apenas lesões leves, aqueles com pelo menos uma lesão moderada corresponderam a $24,2 \%$ da população estudada (IC 95\%: 18,5 - 30,0), enquanto os que apresentaram pelo menos uma lesão grave foram 10,7\% (IC 95\%: 6,6 - |4,8).

Lesões em via aérea inferior (56/507) foram representadas por erosões $(23,2 \%$ das lesões nessa região), ulcerações (17,9\%), traqueíte e/ou bronquite $(55,4 \%)$, granuloma em brônquio principal direito $(1,8 \%)$ e estenose do brônquio principal direito (I,8\%).

$\mathrm{Na}$ Tabela 2 descrevemos os tipos de lesões encontradas por faixa etária e gravidade. Erosões foram as lesões que apresentaram maior incidência em ambos os grupos etários, tendo representado 40,5\% das lesões em crianças fora do período neonatal e 37,6\% daquelas em recém-nascidos $(p=0,61)$ e ocorreram com maior incidência na subglote para as crianças maiores de 28 dias de vida e de forma similar na glote e subglote nos recém-nascidos. 
Tabela 2-Achados endoscópicos em 215 pacientes de acordo com a localização anatômica e gravidade da lesão e por faixa etária

\begin{tabular}{|c|c|c|c|c|c|c|c|c|}
\hline \multirow[t]{2}{*}{ Prognóstico } & \multicolumn{2}{|c|}{$\begin{array}{l}\text { Supraglote } \\
(n=30)\end{array}$} & \multicolumn{2}{|c|}{$\begin{array}{l}\text { Glote } \\
(n=244)\end{array}$} & \multicolumn{2}{|c|}{$\begin{array}{l}\text { Subglote } \\
(n=177)\end{array}$} & \multicolumn{2}{|c|}{$\begin{array}{l}\text { TOTAL } \\
(n=45 I)\end{array}$} \\
\hline & $>28 \mathrm{~d}$ & $\leq 28 \mathrm{~d}$ & $>28 \mathrm{~d}$ & $\leq 28 \mathrm{~d}$ & $>28 d$ & $\leq 28 \mathrm{~d}$ & $>28 d$ & $\leq 28 \mathrm{~d}$ \\
\hline \multicolumn{9}{|l|}{ LEVES } \\
\hline Edema & | & 0 & 9 & 7 & 4 & 2 & 14 & 9 \\
\hline Edema + Hiperemia & 19 & 8 & 2 & 2 & 27 & 7 & 48 & 17 \\
\hline Erosão & 0 & 0 & 65 & 30 & 80 & 26 & 145 & 56 \\
\hline Hiperemia & 0 & 2 & I & 3 & 4 & 0 & 5 & 5 \\
\hline Subtotal & 20 & 10 & 77 & 42 & 115 & 35 & 212 & 87 \\
\hline \multicolumn{9}{|l|}{ MODERADAS } \\
\hline Edema de PV & & - & 48 & 19 & - & . & 48 & 19 \\
\hline Ulceracãa & 0 & 0 & 16 & 8 & 9 & 4 & 25 & 12 \\
\hline Tecido de granulação & 0 & 0 & 7 & 3 & 0 & 0 & 7 & 3 \\
\hline Exposição cartilagem. & 0 & 0 & I & 0 & 3 & 2 & 4 & 2 \\
\hline Lesão de PV & & - & I & 2 & - & - & I & 2 \\
\hline Subtotal & 0 & 0 & 73 & 32 & 12 & 6 & 85 & 38 \\
\hline \multicolumn{9}{|l|}{ GRAVES } \\
\hline Granuloma & 0 & 0 & 0 & 0 & I & 0 & I & 0 \\
\hline Sinéquia & 0 & 0 & 5 & 2 & 1 & I & 6 & 3 \\
\hline Estenose & 0 & 0 & 0 & 0 & 3 & 3 & 3 & 3 \\
\hline Nódulos Fibrosos PV & - & - & 6 & 3 & - & - & 6 & 3 \\
\hline Paresia PV & - & - & 0 & I & - & - & 0 & I \\
\hline Sulcos cicatriciais PrVcAr & & & 3 & 0 & & & 3 & 0 \\
\hline Subtotal & 0 & 0 & 14 & 6 & 5 & 4 & 19 & 10 \\
\hline $\begin{array}{l}\text { TOTAL DE LESÕES } \\
\text { EM VAS E SUBGLOTE }\end{array}$ & 20 & 10 & 164 & 80 & 132 & 45 & 316 & 135 \\
\hline
\end{tabular}

VAS- via aérea superior; PV- prega vocal; PrVcAr- processos vocais das aritenóides

Dentre as lesões classificadas como moderadas observamos maior incidência de edema em pregas vocais em ambos os grupos ( $13,4 \%$ das lesões em crianças maiores de 28 dias de vida e $12,8 \%$ das lesões em recém-nascidos) $(p=0,96)$.

Lesões graves ocorreram principalmente ao nível da glote tendo sido $73,7 \%$ das lesões dessa natureza e nessa topografia no grupo etário fora do período neonatal e $60 \%$ em recém-nascidos $(p=0,67)$. Sinéquia foi a lesão grave de maior incidência em crianças maiores de 28 dias de vida ( $1,7 \%$ do total de lesões) assim como nódulos fibrosos em pregas vocais ( $1,7 \%$ do total de lesões). Para os recém-nascidos sinéqüias, estenoses e nódulos fibrosos em pregas vocais apresentaram incidência similar (2\% do total de lesões para cada). Estenose subglótica apresentou uma incidência de 2,8\% na população estudada (6/215 sendo três pacientes recém-nascidos e três pertencentes ao grupo fora do período neonatal).
Foram registrados em vídeo 50 exames, sendo 44 em crianças maiores que 28 dias de vida e seis em recém-nascidos $(p=0,86)$. A amostra analisada foi composta por cinco exames normais e 45 exames alterados onde foram diagnosticadas 90 lesões. $O$ prognóstico das lesões em questão apresentou um perfil similar ao do conjunto das lesões. Lesões leves representaram 60\% da amostra, moderadas representaram $21,1 \%$, graves foram $5,6 \%$ da amostra e outras lesões foram $13,3 \%$. Com relação aos achados positivos houve $100 \%$ de concordância entre os observadores. Houve discordância em relação a cinco exames (10\%) com relação a achados não relacionados à intubação e às lesões moderadas. A taxa geral de concordância foi de 84\% (IC 95\%: 74 - 94\%).

\section{Discussão}

Alguma lesão em via aérea de pacientes submetidos à intubação é um achado esperado em função do conhecimento da fisiopatologia dessas lesões. O desenvolvimento de lesões de maior gravidade sofre possivelmente a influência de variáveis relativas ao paciente e ao procedimento de intubação. Aquelas relativas ao procedimento são de grande interesse, pois são passíveis de intervenção. Para tal é essencial o conhecimento da incidência desses eventos.

Observamos uma elevada incidência (89,9\% da população estudada) de lesões em vias aéreas secundárias à intubação traqueal, de gravidades variadas. A taxa geral de concordância entre os dois observadores sugere que os resultados demonstrados devem estar próximos à realidade com mínima ocorrência de um viés de observação. Uma vez que reavaliação endoscópica foi realizada apenas para os pacientes que necessitaram reintubação, é possível que a incidência de lesões graves tenha sido subestimada. O processo de reparação que se inicia após o trauma se completa após a retirada do tubo podendo durar até semanas. Dessa maneira é possível que dentre aqueles não reavaliados, alguns tenham evoluído com lesões graves em via aérea. Essa possibilidade teria então interferido no cálculo de incidência dessas lesões que poderiam estar subestimadas. Para as lesões classificadas como moderadas essa eventualidade é mais remota, uma vez que lesões leves evoluem para formas moderadas apenas com a persistência da agressão. Já as moderadas podem evoluir para resolução, ou para lesões graves como formação de sinéquia ou estenose após a retirada do tubo.

Fan et al. ${ }^{14}$, em estudo de 95 recémnascidos submetidos a intubação traqueal, observaram exame normal em apenas 19\%. Com relação aos pacientes com exames alterados, os autores adotaram classificação segundo a qual lesões menores, tais como ulceração na região interaritenóide, pregas vocais e subglote, granulomas em pregas vocais e paresia transitória das pregas, não eram associadas à obstrução da via aérea. As lesões moderadas cursavam com algum grau de obstrução, e incluíam edema, pseudomembranas e granulomas maiores, associados ou não a lesões menores. Lesões maiores corresponderam à obstrução fixa e grave, geralmente decorrente de estenose subglótica, membrana subglótica, defeitos em pregas vocais e estenose traqueal. Os resultados desse estudo mostraram que $40 \%$ dos pacientes apresentaram lesões menores, 34\% lesões 
moderadas e $9 \%$ lesões maiores. Esses achados são muito similares aos por nós observados tanto em termos de incidência, quanto na distribuição da gravidade das lesões. No entanto, as classificações adotadas foram distintas, e no estudo de Fan et al. ${ }^{14}$ os autores estudaram apenas recém-nascidos, enquanto no presente estudo incluímos também crianças fora do período neonatal.

É possível estabelecer um paralelo com a incidência de estenose subglótica que foi por nós classificada como lesão grave e diagnosticada em seis pacientes (2,8\%). A incidência reportada de estenose subglótica experimentou uma redução nos últimos 30 anos. Estudos conduzidos nos anos 70 mostravam uma incidência de 3,8\% a 8,3\%8,15,22-25. Aqueles realizados posteriormente mostraram ausência de estenose subglótica em algumas séries $^{26,27}$ ou baixa incidência em outros estudos $(0,4 \%-3,2 \%)^{10,11,13,14,28,29}$. Relatos com elevadas incidências dessa forma de lesão ( $12,8 \%$ $24,5 \%$ ) são provavelmente decorrentes das características da população estudada (recémnascidos de muito baixo peso ao nascimento) e pela maior sobrevida desses pacientes ${ }^{9,12}$. Os estudos identificados se referem a uma população específica de recém-nascidos prematuros. A menor incidência de estenose subglótica nos recém-nascidos incluídos no presente estudo pode ter sido decorrente do fato de a nossa população não ter sido fortemente representada por neonatos prematuros de muito baixo peso (média do peso a extubação $=2.539,4 \pm 833,3$; variação $=1.250$ $4.600 \mathrm{~g})$. Nos demais estudos o peso de nascimento médio foi de 1.229,7 \pm 137,3 gramas com variação de 496 a $4.850 \mathrm{~g}^{9 \cdot 14}$. A prematuridade extrema pressupõe uma maior duração da intubação com maior risco de exposição às demais variáveis de risco, além da imaturidade do sistema respiratório.

Outro aspecto que dificulta comparações é o denominador a partir do qual a incidência de estenose subglótica é reportada. Alguns autores reportam seus dados a partir do número de recém-nascidos admitidos enquanto outros reportam a partir do número de recém-nascidos submetidos à intubação traqueal, e ainda a partir do número de pacientes intubados por determinado período $9,11,12$. Incidência de 4,9\% de estenose subglótica em recém-nascidos encontrada na nossa pesquisa teve como denominador todos os recém-nascidos intubados, exceto aqueles com peso à extubação inferior a I.250g. Podemos perceber que as populações estudadas são distintas, o que permite apenas inferir similaridades ou diferenças entre os resultados apresentados relativos à estenose subglótica.

Encontramos uma maior incidência de lesões na região da glote ( $48,1 \%$ das lesões) e subglote $(35,1 \%$ das lesões) sem diferença estatisticamente significante entre os grupos etários com relação aos achados em glote e subglote, mas com diferença significante $(p<0,00 \mathrm{I})$ quando comparamos as duas regiões, tendo sido mais freqüente 0 acometimento da glote independente da faixa etária. Esses resultados mostraram maior acometimento da via aérea superior, em particular nas regiões posteriores da glote.

Acometimento da via aérea abaixo da região subglótica é descrito principalmente em estudos envolvendo pacientes adultos, nos quais existe uma grande preocupação com lesões induzidas pelo balonete do tubo traqueal. Para a faixa etária pediátrica, a fisiopatologia das lesões em via aérea inferior está principalmente relacionada a trauma pela extremidade do tubo ou traumatismo da mucosa em decorrência de aspirações do tubo traqueal, uma vez que tubos com balonete são raramente utilizados. Lesões do tipo traqueíte e/ou bronquite não estão diretamente relacionadas ao tubo traqueal, mas provavelmente à pressão positiva do respirador, concentração de oxigênio, umidade e temperatura do ar inspirado ${ }^{30}$. A baixa incidência de lesões reportadas abaixo da subglote pode ser devida a viés de observação uma vez que os autores pesquisados se concentraram em reportar a incidência de estenose subglótica ou pelo fato de que tubos sem balonete são geralmente recomendados para crianças menores de oito a 10 anos $^{31}$. Essa recomendação se baseia nos conhecimentos da anatomia da via aérea nesta faixa etária. A região subglótica que corresponde ao anel da cartilagem cricóide representa o ponto de maior estreitamento da via aérea, atuando como "balonete funcional". No entanto, essa prática tem sido revista em função de estudos que mostraram incidência de estridor pós-extubação similar entre pacientes que utilizaram tubos com e sem balonete ${ }^{32,33}$.

O desenvolvimento de lesões secundárias à intubação é certamente um fenômeno multifatorial, desse modo diversas variáveis podem ter contribuído para a elevada incidência de alguma lesão observada nessa população. O diâmetro do tubo traqueal é freqüentemente citado como fator de risco para lesões ${ }^{6,9,12}$. Identificamos uma variedade de propostas para a escolha do diâmetro do tubo traqueal em crianças, tais como o diâmetro do dedo mínimo, diâmetro do leito ungueal do dedo mínimo, fórmulas baseadas na idade ou estimativas de peso a partir da estatura ${ }^{34-38}$. No presente estudo a escolha do diâmetro do tubo traqueal foi baseada em fórmulas estruturadas no peso e idade com possibilidade de uso de tubos com diâmetros $0,5 \mathrm{~mm}$ maior ou menor do que os definidos pelas propostas $^{31}$. A precisão dessas recomendações foi contestada anteriormente ${ }^{37}$. Dessa maneira, uma postura prudente é aquela de se basear em alguma recomendação e procurar utilizar o tubo de menor diâmetro possível que permita um escape e a adequada ventilação. De fato, o escape é recomendável e deve estar presente com a aplicação de uma pressão positiva de 20-30 $\mathrm{cm} \mathrm{H}_{2} \mathrm{O}^{38}$. Apesar de termos baseado a escolha do tubo traqueal em fórmulas propostas na literatura, não é possível descartar que tenha ocorrido inadequação do diâmetro, pois não foi aferida a presença de escape. Apesar dessa possibilidade a incidência de lesões graves, em particular da estenose subglótica, foi comparável aos estudos publicados.

\section{Conclusões}

Nessa população de 215 pacientes observamos elevada incidência de lesões em vias aéreas secundárias à intubação traqueal, sem diferença estatisticamente significante entre os grupos etários para nenhuma das características estudadas. Houve predomínio de lesões classificadas como leves, de acometimento da glote e lesões caracterizadas por erosão, edema e ulceração. No entanto, lesões moderadas e graves estiveram presentes. O conhecimento da distribuição das lesões quanto à gravidade e localização anatômica pode auxiliar na redução de lesões com potencial de evolução desfavorável bem como das seqüelas de intubação. Estudos de acompanhamento clínico e/ou endoscópico de pacientes com lesões moderadas à extubação são necessários para definir o prognóstico associado a essa forma de lesão. 


\section{SUMMARY}

\section{INCIDENCE AND ENDOSCOPIC CHARAC- TERISTICS OF AIRWAY INJURIES ASSOCIATED WITH ENDOTRACHEAL INTUBATION IN CHILDREN}

ObJECTIVE, describe the incidence and endoscopic characteristics of airway injuries in children submitted to intubation.

Methods. during a two-year period (octoberl 1999-october/2001) we conducted a prospective study in which all patients that required intubation, excluding those who deceased before extubation and newborns (NB) weighing less than $1.250 \mathrm{~g}$, were submitted to airway endoscopy at extubation. The endoscopic findings were classified as minor, moderate or severe. Descriptions were made through proportions and medians, comparisons were done through chi-square for proportions.

RESULTS. we studied 6 I NB and 154 children. In 89.8\%, $55 \mathrm{NB}$ and 138 children ( $P=0.89)$, it was detected at least one lesion in a total of 507. Patients with minor lesions were $54.8 \%$ (IC95\%:48. I-6I.5), those with moderate were 24.2\% (IC95\%:18.5-30.0) and severe injury occurred in 10.7\% (IC95\%:6.6-14.8). Lesions at the glottis (48.1\% of lesions) and subglottis (35.1\%) presented the highest incidence. Erosions had the highest incidence in both age groups $(P=0.88)$. Vocal folds edema was the main moderate lesion in both groups $(P=0.96)$, followed by ulcerations $(P=0.92)$. Fibrous nodules at vocal folds and adhesions were the main severe injuries in both groups $(P=0.12)$. Subglottic stenosis was detected in $2.8 \%$ of the patients without difference between groups $(P=0.35)$.

Conclusions. we observed a high incidence of airway injury, without statistical significant difference between age groups in regard to the incidence and characteristics of the injuries. Minor injury was detected in the majority of the population. Lesions were mainly noticed at the glottis and were characterized by erosions, edema and ulcerations. [Rev Assoc Med Bras 2004; 50(I): 87-92]

Key words: Intratracheal intubation. Child. Newborn. Airway injury. Subglottic stenosis.

\section{REFERÊNCIAS}

I. Nilsson E. On treatment of barbiturate poisoning. Acta Med Scand 195I; 253:7- I 27.

2. Dwyer CS, Kronenberg S, Saklad M. The endotracheal tube: a consideration of its traumatic effects with a suggestion for the modification thereof. Anesthesiology 1949; 10:7|4-28.

3. Donnelly WH. Histopathology of endotracheal intubation. An autopsy study of 99 cases. Arch Pathol 1969; 88:5।I-20.

4. Joshi W, Mandavia SG, Stern L, Wiglesworth FW. Acute lesions induced by endotracheal intubation. Occurrence in the upper respiratory tract of newborn infants with Respiratory Distress Syndrome. Am J Dis Child 1972; 124:646-9.

5. Bishop MJ, Weymuller EA Jr, Fink BR. Laryngeal effects of prolonged intubation. Anesth Analg 1984; 63:335-42.

6. Benjamin B. Laryngeal trauma from intubation: endoscopic evaluation and classification. In: Cummings CW, Fredrickson JM, Harker LA, Krause C], Schuller DE, editors. Otolaryngology head and neck surgery. 2nd ed. St Louis: Mosby-Year Book; 1993. p. 1875-96.

7. Weymuller EA Jr, Bishop MJ, Fink BR, Hibbard AW, Spelman FA. Quantification of intralaryngeal pressure exerted by endotracheal tubes. Ann Otol Rhinol Laryngol 1983; $92(5$ pt I):444-7.

8. Choffat, JM, Goumaz CF, Guex JC. Laryngotracheal damage after prolonged intubation in the newborn infant. In: Stetson JB, Sawyer RR, editors. Neonatal intensive care. St. Louis: Mosby Year Book: 1976. p.253-70.

9. Sherman JM, Lowitt S, Stephenson C, Ironson, G. Factors influencing acquired subglottic stenosis in infants. J Pediatr 1986; 109:322-7.

10. Nicklaus PJ, Crysdale WS, Conley S, White AK, Sendi K, Forte V. Evaluation of neonatal subglottic stenosis: a 3-year prospective study. Laryngoscope 1990; 100: 1185-90.

11. Grundfast KM, Camilon FS Jr, Pransky S, Barber CS, Fink R. Prospective study of subglottic stenosis in intubated neonates. Ann Otol Rhinol Laryngol 1990; 99(5 pt I): 390-5.

12. Downing G], Kilbride HW. Evaluation of airway complication in high-risk preterm infants: application of flexible airway endoscopy. Pediatrics 1995; 95:567-72.

13. Silva O, Stevens D. Complications of airway management in very-low-birth-weight infants. Biol Neonate 1999; 75:40-5.

14. Fan LL, Flynn JW, Pathak DR. Risk factors predicting laryngeal injury in intubated neonates. Crit Care Med 1983; 1 1:431-3.

15. Strong RM, Passy V. Endotracheal intubation. Complications in neonates. Arch Otolaryngol 1977; 103:329-35.

16. Hawkins DB. Glottic and subglottic stenosis from endotracheal intubation. Laryngoscope 1977; 87:339-46.

17. Blahova O, Brezovsky P. Stenosing processes due to endotracheal intubation and tracheostomy in children. Int J Pediatr Otorhinolaryngol 1981; 3:199-203.

18. Volpi D, Lin PT, Kuriloff DB, Kimmelman CP. Risk factors for intubation injury of the larynx. Ann Otol Rhinol Laryngol 1987; 96:684-6.

19. Woo P, Kelly G, Kirshner P. Airway complications in the head injured. Laryngoscope 1989; $99(7 \mathrm{pt} \mathrm{I):725-31.}$

20. Thomas R, Kumar EV, Kameswaran M, Shamin A, Al Ghamdi S, Mummigatty AP, et al. Post intubation laryngeal sequelae in an intensive care unit. J Laryngol Otol 1995; 109:3 I3-6.

21. Pollack MM, Ruttimann UE, Getson PR. Pediatric Risk of Mortality (PRISM) Score. Crit
Care Med 1988; 16:1110-6.

22. Freeman GR. A comparative analysis of endotracheal intubation in neonates, children and adults: complications, prevention and treatment. Laryngoscope 1972; 82: I385-98.

23. Parkin JL, Stevens MH, Jung AL. Acquired and congenital subglottic stenosis in the infant. Ann Otol Rhinol Laryngol 1976; 85 (pt I): 573-81.

24. Jones R, Bodnar A, Roan $Y$, Johnson D. Subglottic stenosis in newborn intensive care unit graduates. Am J Dis Child 1981; 135:367-8.

25. Papsidero MJ, Pashley NR. Acquired stenosis of the upper airway in neonates. An increasing problem. Ann Otol Rhinol Laryngol 1980; 89 (pt I):5।2-4.

26. Contencin P, Narcy P. Size of endotracheal tube and neonatal acquired subglottic stenosis. Study Group for Neonatology and Pediatric Emergencies in the Parisian Area. Arch Otolaryngol Head Neck Surg 1993; I 9 9:8 I 5-9.

27. Walner DL, Loewen MS, Kimura RE. Neonatal subglottic stenosis-incidence and trends. Laryngoscope 200 I; I1 1:48-51.

28. Dankle SK, Schuller DE, McClead RE. Risk factors for neonatal acquired subglottic stenosis. Ann Otol Rhinol Laryngol 1986; 95 (pt 1):626-30.

29. Ratner I, Whitfield J. Acquired subglottic stenosis in the very-low birth-weight infant. Am J Dis Child 1983; 137:40-3.

30. Turner BS, Loan LA. Tracheobronchial trauma associated with airway management in neonates. AACN Clin Issues 2000; 11:283-99.

31. Chameides L, Hazinski MF. Recognition of respiratory failure and shock. Pediatric advanced life support. Dallas: AHA; 1997. p.2.2-2.10.

32. Deakers TW, Reynolds G, Stretton M, Newth C). Cuffed endotracheal tubes in pediatric intensive care. J Pediatr 1994; 125:57-62.

33. Khine HH, Corddry DH, Kettrick RG, Martin TM, McCloskey JJ, Rose JB, et al. Comparison of cuffed and uncuffed endotracheal tubes in young children during general anesthesia. Anesthesiology 1997; 86:627-31.

34. King BR, Baker MD, Braitman LE, SeidlFriedman J, Schreiner MS. Endotracheal tube selection in children: a comparison of four methods. Ann Emerg Med 1993; 22:530-4.

35. Van den Berg AA, Mphanza T. Choice of tracheal tube size for children: finger size or agerelated formula? Anaesthesia 1997; 52:70 I-3.

36. Hofer CK, Ganter M, Tucci M, Klaghofer R, Zollinger A. How reliable is length-based determination of body weight and tracheal tube size in the paediatric age group? The Broselow tape reconsidered. Br J Anaesth 2002; 88:283-5.

37. Eck JB, De Lisle DG, Phillips-Bute BG, Ginsberg B. Prediction of tracheal tube size in children using multiple variables. Paediatr Anaesth 2002, 12:495-8.

38. Zaritsky AL, Nadkari VM, Hickey RW, Schexnayder SM, Berg RA. Airway, ventilation and management of respiratory distress and failure. Pediatric advanced life support. Dallas: AHA; 2002. p.8I- 126.

Artigo recebido: I I/02/03

Aceito para publicação: 03/06/03 